\title{
Surfaces
}

\section{LE CINÉMA COMME OPÉRATEUR D’ANALYSE}

\section{Réda Bensmaïa}

Volume 1, 1991

URI : https://id.erudit.org/iderudit/1065251ar

DOI : https://doi.org/10.7202/1065251ar

Aller au sommaire du numéro

Éditeur(s)

Les Presses de l’Université de Montréal

ISSN

1188-2492 (imprimé)

1200-5320 (numérique)

Découvrir la revue

Citer cet article

Bensmaïa, R. (1991). LE CINÉMA COMME OPÉRATEUR D’ANALYSE. Surfaces, 1. https://doi.org/10.7202/1065251ar
Résumé de l'article

Comment le film, d'abord objet d'analyse, est devenu un modèle d'analyse. Le film et la nature disciplinaire de la théorie.
Copyright @ Réda Bensmaïa, 1991

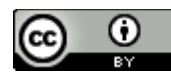

Ce document est protégé par la loi sur le droit d'auteur. L’utilisation des services d'Érudit (y compris la reproduction) est assujettie à sa politique d'utilisation que vous pouvez consulter en ligne.

https://apropos.erudit.org/fr/usagers/politique-dutilisation/ 


\title{
LE CINÉMA
}

\section{COMME OPÉRATEUR D'ANALYSE}

$\underline{\text { Réda Bensmaïa }}$

\section{RÉSUMÉ}

Comment le film, d'abord objet d'analyse, est devenu un modèle d'analyse. Le film et la nature disciplinaire de la théorie.

\begin{abstract}
How film has moved from being an object of analysis to being a model for analysis. Film and the disciplinary nature of theory.
\end{abstract}

$<<$ Quelle évaluation peut-on porter sur la notion d'opérateur appliquée au cinéma? >> Autrement dit: <<De quelle(s) manière(s) la mise en avant du film comme $<<$ modèle $>>$ d'analyse a-t-elle affecté la réflexion épistémologique sur les disciplines théoriques autres que la théorie du cinéma? >> Pour aborder ces <<questions transversales $>>$, il m'a paru indispensable de commencer par un détour qui permette d'inscrire la notion d'opérateur dans un cadre historique et critique où elle n'apparaîtrait plus comme un phénomène qui se serait greffé de manière arbitraire sur les théories du cinéma existantes, mais au contraire comme l'instrument d'une reprise critique inévitable. Pour ce faire, il m'a paru indispensable 
d'esquisser une carte du terrain théorique où cette notion a fini par s'imposer historiquement. Pour qui s'est un tant soit peu familiarisé avec la critique et la théorie modernes du cinéma, s'il y a une chose qui frappe c'est bien évidemment la très grande variété de formes et de discours qui les ont pris en charge. Critique qui est allée de la simple recension de journal aux analyses détaillées que l'on allait trouver dans les magazines de cinéma. Ou $<<$ théorie $>>$ qui, de simple $<<$ esthétique $>>$ appliquée (au cinéma), allait bientôt se présenter comme un $<<$ corps de concepts $>>$ cinématographiques sui generis [1] . D'une /pp 5-6/ manière plus générale, on ne manquera pas non plus d'être frappé par la diversité et la sophistication, extrêmement poussée parfois, des théories du cinéma que l'on peut trouver de nos jours dans les revues spécialisées. Cette dernière tendance en est au point où, pour l'amateur de cinéma et des arts audiovisuels, le cinéma est devenu absolument indissociable des discours $<<$ techniques $>>$ ou $<<$ techno-logiques $>>$ qui lui sont associés ainsi que de la $<<$ critique $>>$ de ces $<<$ discours $>>$. Aujourd'hui, le véritable $<<$ amateur $>>$ de cinéma, tout comme le cinéaste, ne se voudra plus un $<<$ esthète $>>$ ou un simple $<<$ cinéphile $>>$, mais un $<<$ théoricien $>>$ : il ne va plus au cinéma pour ajouter un film à son palmarès, mais pour confronter sa pensée-cinéma au texte filmique; il ne sort pas du cinéma pour renouer immédiatement avec le monde, mais en se posant la question de savoir si le film qu'il a vu est $<<$ synchrone $>>$ ou s'il l'a aidé à $<<$ croire encore au monde $>>[2]$. Toujours est-il qu'un regard plus /pp 6-7/

analytique sur ce qu'est devenu le rapport que nous avons au cinéma y décèlera un changement de nature radicale: nous n'en sommes plus aujourd'hui à l'époque où des esprits éclairés[3] pouvaient proposer des articles ou des livres sur le cinéma, sans que cela ne soulève un réel problème théorique. Lorsque Bazin écrivait les articles qui allaient constituer Qu'est-ce que le Cinéma?, lorsque Balàsz publiait sa Theory of the Film ou encore lorsque Élie Faure écrivait les textes qui allaient former sa Fonction du cinéma nous étions encore à des années-lumière de la période où de simple prétexte à des spéculations esthétiques ou idéologiques, le cinéma allait comme soudainement devenir un $<<$ objet $>>$ de recherche $<<$ scientifique >>: soit l'objet de discours qui voudront l'arracher à $l^{\prime}<<$ impressionnisme $>>$, critique dont il avait illégitimement été la proie. Tels ont été à coup sûr les objectifs des courants critiques qui ont abouti à la création de la Filmologie, puis de la Sémiologie du cinéma, puis de l'analyse textuelle, etc.[4]

Dans une telle perspective, l'époque $<<$ Élie Faure $>>$ [5] peut nous paraître comme l'époque $<<$ heureuse $>>$ (Barthes) durant laquelle, s'il est l'objet d'un émerveillement croissant, le cinéma ne fait /pp 7-8/ pas encore $<<$ problème $>>$. C'est plutôt une $<<$ époque $>>$ [6] où le cinéma semble encore consentir à faire corps avec les critiques $<<$ idéologiques $>>$, $<<$ esthétiques $>>$ ou $<<$ sémiologiques $>>$ auxquelles on le soumet, et ne point exiger de $<<$ théorie $>>$. 
Au départ, parce qu'il est considéré comme un art populaire ou de masse, le cinéma s'est volontiers prêté à la multiplicité des discours $<<$ théoriques $>>$ qui le prenaient en charge pour en vendre les produits, en vanter les mérites, en critiquer la médiocrité ou en saluer la modernité. Mais ce qui est important de relever après avoir fait ce constat, c'est que ce n'est que dans un second temps qu'une critique et bientôt une théorie allaient $\mathrm{s}^{\prime}<<$ imposer $>>$ à lui et le constituer comme $<<$ objet $>>$. Ce n'est donc qu'après-coup -- soit après que le cinéma se soit révélé plus revêche à la $<<$ réduction $>>$ conceptuelle qu'on ne l'avait tout d'abord imaginé -- qu'on a consenti à ne plus se contenter de le débiter en tranches sémantiques ou formelles pour tenter avec des moyens renouvelés de mettre en évidence, et /pp 8-9/bientôt d'en démonter, la $<<$ spécificité $>>$ [7]. L'époque des $<<$ observateurs avertis $>>$, et bientôt des $<<$ théoriciens $>>$ du cinéma, n'allait plus tarder à s'annoncer: $<<$ Qu'il s'agisse d'un bon ou d'un mauvais film, d'une production romancée, scientifique ou documentaire, écrivait déjà Élie Faure dans un texte de 1934, un observateur averti ne peut se défendre d'y découvrir les éléments caractéristiques d'un art absolument original. $>>$ [8] Or c'est précisément cette $<<$ originalité $>>$ - -- rebaptisée $<$ <pécificité>> par la sémiologie du cinéma -- qui me semble jouer ici le rôle le plus important. C'est en tous cas cette idée de l'originalité du cinéma qui ne cessera d'orienter les travaux qui lui seront dorénavant consacrés.

En effet, si le cinématographe des frères Lumière suscite l'intérêt des scientifiques et celui de Méliès celui des foules, sa métamorphose en cinéma cinéma, elle, crée une situation radicalement nouvelle. Il ne s'agira plus désormais d'en demander plus aux créateurs comme Méliès ou Griffith ou de militer pour que des transformations techniques viennent améliorer les $<<$ performances $>>$ de cet instrument $<<$ polymorphe $>>$ et versatile. Il ne s'agira pas non plus de renchérir sur la moralité ou l'immoralité, le réalisme ou l'irréalisme, la $<<$ transparence $>>$ ou l'<<opacité $>>$ de ce médium extraordinaire[9]:/pp 9-10/ désormais le cinéma subira une mutation qui le transformera en cette $<<$ machine de vision $>>$ qui $<<$ dédouble $>>$ les points de vue et rend problématique le $<<$ partage de la perception de l'environnement entre l'animé, le sujet vivant, et l'inanimé $>>[10]$ et tend de plus en plus à devenir un $<<$ modèle $>>$ (d'analyse) de la pensée plutôt qu'un $<$ <objet>> pour une pensée déjà donnée: $<<$ Le problème n'est donc plus tellement celui des seules images mentales de la conscience [et, ajouteronsnous, celui de son $<<$ adéquation $>>$ à l' $<<$ ambiguïté $>>$ du réel (selon un Bazin) ou à la morphologie du langage (Metz)], mais plutôt celui des images virtuelles instrumentales de la science et de leur caractère paradoxalement factuel $>>$ [11]. Ce n'est donc plus notre savoir (linguistique, esthétique ou sémiotique) qui <<informe>> le cinéma, mais bientôt le penser-cinéma et, pourrait-on dire, l'agencer-cinéma [12] qui se met à nous informer sur les modalités de notre rapport au monde: montage, décadrage, coupures irrationnelles, images pures du temps $(<<$ cristaux $>>)$, mémoire, nappes de temps et multiplicités paradoxales[13]. C'est dire que le rapport au /pp 10-11/ cinéma change totalement de nature: il s'agira moins désormais de rapporter des images (représentatives ou pas) à un langage que de confronter nos régimes symboliques et les modalités de notre penser, 
fantasmer, désirer -- voire même interpréter - -- à la logique, pourrait-on dire, spontanément déconstructive du cinéma. La question qui se posera à ces amateurs particuliers que sont les théoriciens $d u$ cinéma sera dorénavant une question beaucoup plus radicale. Ce sera bien une fois encore la question $<<$ Qu'est-ce que le cinéma? $>>$, mais plus du tout à la manière ontologique d'André Bazin. C'est que $l^{\prime}<<$ objet $>>$ en question ne sera plus la représentation ( $\mathrm{du}<<$ réel $>>$ ) ou la teneur symbolique des images, mais celui de l'essence de la $<<$ machine $>>$ qui leur donne lieu comme effets de son fonctionnement. Avec le recul dont nous disposons aujourd'hui, on pourrait dire que la théorie du cinéma est née de l'insatiable désir de la critique à vouloir délimiter son $<<$ objet $>>$ pour rendre compte de son $<<$ originalité $>>$ et de sa $<<$ spécificité $>>$. Mais très vite une autre difficulté est venue se greffer sur cette entreprise: celle de vouloir séparer l'analyse de l'image (cinégraphique) et de ses $<<$ parties $>>$ de l'analyse de la langue: $<<$ Quelle partie de l'image, quelle partie de la langue?>> [14]. Le mot d'ordre qui allait bouleverser la théorie du cinéma était lancé: il s'agissait dorénavant de dé-limiter, de couper à même la chair de l'image pour y voir clair et pouvoir en découdre avec le cinéma comme langage spécifique. Et pour un temps, il parut aux chercheurs que ce n'était qu'au prix de ce $<<$ transfert $>>$, ou peut-être mieux de cette $<<$ translation $>>$, qu'une $<<$ science du cinéma $>>$ devenait possible. À quelle $<<$ exigence $>>$ correspond un tel $<<$ découpage $>>$ de l'image? À quelle nécessité épistémologique le partage, ou peut-/pp 11-12/ être mieux la partition, entre l'image et la langue $<<$ linguistique $>>$ qui nous est proposé ici renvoyait-il? De quelle manière annonçait-il les autres ruptures que nous avons enregistrées depuis? C'est en essayant de répondre à ces questions que l'on pourra peut-être comprendre la nature de la $<<$ mutation $>>$ dont nous parlions plus haut et des enjeux théoriques qui l'ont accompagnée.

La première remarque que l'on peut faire à propos du rapport de la théorie au cinéma, c'est que, comme nous l'avons suggéré plus haut, sous sa forme moderne la théorie est un produit relativement tardif. En effet, pendant près d'un demi-siècle, les courants dominants de la critique cinématographique ne se sont pas réclamés d'une $<<$ théorie $>>$ spécifique. Si, par $<<$ théorie $>>$, on entend l'ancrage de l'analyse et de l'évaluation critique dans un système conceptuel homogène, aucun des grands critiques de cinéma ne peut être considéré - -- ou ne s'est considéré -- comme un $<<$ théoricien $>>$ à proprement parler. Rien en tous cas dans leurs travaux ne se présentait comme quelque chose à prendre en bloc ou à laisser.[15] Et en ce sens, on peut dire que nous avions affaire à des théories $<<$ faibles $>>$ [16]. Tous ont bien recours à un minimum de conceptualisation, mais aucun n'exhibe l'assurance qu'affiche ce qu'on appelera la $<<$ théorie >> du cinéma. S'il y a déjà des polémiques à l'époque d'Élie Faure ou de Bazin, et à plus forte raison à l'époque de Balàsz ou de Kracauer -- des polémiques qui reposent sur des choix /pp 12-13/ esthétiques, politiques ou philosophiques différents --, jamais cela ne mènera à une remise en question de la compétence de ces critiques ou du bien-fondé de leurs travaux. Chacun d'entre eux se payait la part du lion dans un domaine assez nouveau pour se prêter sans trop de difficultés à tous les discours théoriques possibles: sociologiques, esthétiques, philosophiques, psychologiques, voire même 
religieux. Le meilleur exemple d'une telle tendance -- en France tout au moins -- est celui d'André Bazin, soit celui d'un critique et théoricien qui aura su donner ses lettres de noblesse au cinéma et promouvoir l'image sociale du critique de cinéma en $<<$ honnête homme >> moderne sans entraîner de remue-ménage théorique immédiat.

Or ce qui est frappant ici aussi, c'est qu'à bien considérer les choses, les principes qui avaient présidé à l'éclosion d'une telle ambiance (critique) avaient suivi exactement le même $<<$ mouvement $>>$ et les mêmes principes que ceux qui avaient animé l'évolution de la critique littéraire: soit des $<<$ principes [...] plutôt d'ordre culturel et idéologique que d'ordre proprement théorique, orientés qu'ils étaient plutôt vers l'intégrité d'un Moi social et historique que vers la consistance impersonnelle qui est requise par la théorie>> [17]. Qu'est-ce donc qui, aux alentours des années soixante, est venu si radicalement remettre en question la douceur de ce climat critique pour le transformer en véritable $<<$ champ de bataille $>>$ (théorique)? Ou encore: qu'est-ce qui a donné lieu à la naissance de ce champ encore mal défini que l'on appelle aujourd'hui $<<$ théorie du cinéma >>? Quels <<éléments >> permettent de comprendre les transformations et mutations qui sont venues affecter si radicalement l'étude et le statut du cinéma? Et enfin, qu'est-ce qui, dans ces $<<$ transformations $>>$ et $<<$ mutations $>>$ de la théorie du cinéma, a rendu incontournable /pp 13-14/ la nouvelle (entre)prise théorique qui donne cette fois le cinéma comme opérateur d'analyse?

Pour des raisons qui, je l'espère, apparaîtront de plus en plus clairement par la suite, ce n'est pas chez des historiens ou des théoriciens du cinéma que j'ai trouvé le fil conducteur de mon analyse, mais dans le travail d'un théoricien de la rhétorique: les Allegories of Reading et encore plus Resistance to Theory.de Paul de Man En effet, ce qui m'a frappé en relisant ces textes, c'est l'isomorphie des modalités et des principes qui ont conditionné la formation des théories de la littérature et du cinéma. Si l'on suit le fil conducteur demanien, il apparaît clairement qu'à l'instar de la littérature, la théorie du cinéma ne s'est à son tour imposée comme une $<<$ discipline $>>$ à part que lorsqu'elle a quitté le terrain de l'analyse purement historique ou esthétique, ou encore celui de la signification (idéologique) du cinéma et des films, pour se tourner plutôt vers les modalités de production et de réception de la signification du cinéma à un moment où celles-ci n'étaient pas encore clairement établies[18]: Qu'est-ce que l'essence du cinéma? qu'est-ce qu'un film? qu'est-ce que le langage filmique? peut-on parler d'un texte filmique sans autres formes de procès? Autant de questions aujourd'hui tout à fait $<<$ transparentes $>>$ pour bon nombre de chercheurs, mais qui, il fut un temps, avaient requis la création $\mathrm{d}^{\prime}<<$ une discipline autonome d'investigation critique qui prendrait en considération les /pp 14-15/ possibilités ainsi que le statut théorique de son établissement>> (R.T.,7. C'est moi qui souligne.). 
On connaît certaines des conséquences théoriques que Paul de Man tirera de telles prémisses. La première, c'est que la $<<$ théorie $>>$ de la littérature -- pour nous, celle du cinéma! -- apparaîtra comme contemporaine d'une $<<$ crise $>>$ [19] qui a moins procédé de l'intervention intempestive de telle ou telle discipline <<théorique>> en général -- marxisme, phénoménologie, existentialisme, voire même philologie, psychologie, etc. -- que de l'importation ou du transfert massif des concepts de la linguistique dans la définition et l'analyse de l'objet $<<$ littérature $>>$ et/ou $<<$ cinéma $>>$. Un $<<$ objet $>>$ qui, dans ces conditions, ne s'est plus présenté comme un donné préalable, mais plutôt comme le résultat ou le produit même de l'opération (de transfert) elle-même. Or, comme nous le savons mieux aujourd'hui, cette $<<$ opération>> ne s'est pas faite sans difficultés ni problèmes théoriques. En effet, ce n'est qu'à partir du moment où l'on s'est autorisé [20] à tirer le cinéma du côté des structures du langage /pp 15-16/ que la grande aventure de ce que l'on allait appeler $<<$ théorie du cinéma $>>$ a commencé. Il est vrai qu'il avait fallu entre-temps qu'une autre révolution théorique ait eu lieu, celle qui consistait 1) à considérer le langage comme un <<système autonome $>>$ de signes discrets plutôt que comme un simple instrument de signification et 2 ) à remettre en question la validité des barrières traditionnelles qui existaient entre les usages littéraires et les usages non littéraires -- $<<$ scientifiques $>>$, techniques, etc. -- du langage aux fins de $<<$ libérer le corpus littéraire du poids séculaire de la canonisation textuelle $>>$.[21]

Ce qui allait être décisif dans cette rencontre entre la sémiologie et la littérature -- et, comme nous le pensons, entre la sémiologie et le cinéma --, ce sera le fait qu'à la différence des autres modes d'analyse -- philologique, psychologique, thématique -- <<là où les autres approches étaient dans l'incapacité d'aller au-delà d'observations que l'on pouvait aisément paraphraser ou traduire dans les termes du savoir commun, les analyses [d'inspiration linguistico-sémiologiques] permirent de mettre en évidence des modèles [patterns] qui ne pouvaient cette fois être décrits qu'en des termes spécifiquement linguistiques qui étaient les leurs $>>/ p p$ 16-17/ (Paul de Man, 9). Et de fait, ce qui en littérature allait bientôt être négocié sous la catégorie de $<<$ littérarité $>>$ allait par ailleurs, dans la théorie du cinéma, être subsumé sous la catégorie de (la) <<spécificité $>>$ (du langage cinématographique, par exemple). Ici et là, une rupture allait affecter $l^{\prime}<<$ objet $>>$ (littérature et/ou cinéma) de manière radicale, car si le concept de $<<$ littérarité $>>$ renvoyait à la littérature comme $<<$ le lieu où un savoir en négatif nous est offert quant à la consistance de toutes les propositions linguistiques $>>$ (De Man, 10), celui de <<spécificité $>>$ allait de son côté libérer le cinéma de tout assujettissement à l'impératif de la re-présentation du réel! Dorénavant, et c'est là un $<<$ acquis $>>$ théorique dangereux et aux conséquences immenses, il ne sera plus possible a priori d'affirmer que le langage (ne) fonctionne (que) selon les principes (logiques) qui régissent le monde dit $<<$ phénoménal $>>$. La seule chose que l'on pourra affirmer. mais avec beaucoup de prudence, c'est $<<$ qu'il n'est plus a priori certain que la littérature [et nous ajouterons, le cinéma] puisse être une source fiable d'information sur autre chose que son propre langage $>>$ (De Man, Ibid., 11, C'est moi qui souligne). 
Partie pour arracher la littérature à l'empirisme ou au subjectivisme, la future $<<$ théorie de la littérature $>>$ se retrouvera enfermée dans un cercle vicieux et condamnée à une remise en question perpétuelle de son langage ainsi que des présuppositions théoriques qui avaient présidé à son établissement. Partie à la recherche de son unité et de son unicité théoriques, comme Saul à la recherche de son âne, la théorie de la littérature s'est retrouvée face à un essaim de théories contradictoires qui toutes ont échoué à l'objectiver .[22]/pp 17-18/

Si nous nous tournons vers la théorie du cinéma, il est frappant de constater que l'on a assisté à des ruptures du même type: ici aussi, du fait de l'intervention de la terminologie et de la méthodologie de la linguistique[23], la théorie du cinéma est entrée dans une $<<$ crise $>>$ qui allait prendre, elle aussi, la forme d'une $<<$ résistance $>>$ permanente aux $<<$ théories $>>$ antérieures: une $<<$ résistance $>>$ qu'il ne faudra pas concevoir seulement en termes psychologiques de rejet, mais plutôt en termes fonctionnels et constitutifs de $<<$ fondation $>>$ ! Après la $<<$ première $>>$ sémiologie, il en a fallu une $<<$ seconde $>>$ puis une $<<$ troisième $>>$, non point parce que les deux premières étaient particulièrement déficientes ou fautives, mais de façon plus radicale parce que, comme l'a bien montré Paul de Man, avant d'être une résistance de $l^{\prime}<<$ objet $>>$ (à la théorie), la résistance qui se manifeste dans la théorie a d'abord été une résistance à la lecture ou, peutêtre mieux une résistance à la dimension rhétorique de la lecture.

En effet, ce qui frappe à la relecture de ce qui a été mis en jeu dans cette véritable traversée des régimes signifiants qu'a toujours été ce qu'on a appelé la $<<$ théorie du cinéma $>>$, c'est moins la diversité des $<<$ méthodologies $>>$ ou des registres conceptuels (linguistique, sémiologie, et bientôt psychanalyse, narratologie, théorie du texte, etc.) qui l'a prise en charge, que certaines <<difficultés >> à arraisonner la fondamentale hétérogénéité des régimes de significa-/pp 18-19/tions et de signes qui y était à l'oeuvre. L'autre point important qui va de pair avec le premier, c'est que cette $<<$ percée $>>$ théorique - -- je préfère dire: ce $<<$ coup de force $>>$ théorique! - -- a été lui-même contemporain de la mise en oeuvre d'une techno-logique de l'application et de l'innovation: la linguistique est essentiellement intervenue comme opérateur d'analyse critique des anciennes formes et modalités d'appréhension et de définition du cinéma comme $<<$ objet $>>$ descriptible scientifiquement et avec une rigueur accrue; modalités qu'elle a dû contester et remettre en question constamment pour pouvoir se constituer... en objet !

Forte de ses acquis épistémologiques, elle a pu se présenter comme une discipline qui avait une méthodologie plus $<<$ scientifique $>>$ que les autres méthodes précisément parce qu'elle proposait une définition de son $<<$ objet>> à l'aide de concepts rigoureusement définis --ailleurs!-- et que 
l'on pouvait appliquer. Et ce fut là le moment heureux où, sur de nouvelles bases, le cinéma a de nouveau paru se plier sans trop de difficultés théoriques à la $<<$ machine linguistique $>>$ [24] et faire appel à la création d'une trans-linguistique ou sémiologie. /pp 19-20/

On sait les conséquences théoriques qu'une telle orientation méthodologique a entraînées historiquement. Très vite, elle sera identifiée à la théorie du cinéma elle-même et tout ce que cette dernière comptera comme <<acquis >> véritable lui sera rattaché. Elle ouvrit l'<<époque>> où le discours et les concepts linguistiques sur le cinéma prétendront $<<$ dire littéralement l'objet $>>$. C'est de cette orientation en tous cas que procède la $<<$ rage taxinomique $>>$ qui caractérisera la théorie du cinéma, orientera sa recherche et déterminera la nature de ses $<<$ objets $>>$ : taxinomie des constructions séquentielles $(<<$ Grande syntagmatique $>>)$; taxinomie des différents langages (en termes de $<<$ traits pertinents de l'expression >>); taxinomie des différents types de systèmes et codes du langage cinématographiques; taxinomie des <<enchaînements textuels >> (narratologie)[25],/pp 20-21/ l'un des objectifs étant l'espoir de produire un modèle logico-grammatical qui serait applicable à tout texte (littéraire aussi bien que cinématographique). S'il ne nous appartient pas ici de discuter la validité d'un tel programme méthodologique, il nous est par contre nécessaire de nous interroger sur la nature des présupposés théoriques qui l'ont fondé et qui ont déterminé son orientation spécifique. Ce qui apparait très clairement lorsqu'on aborde ce type de questions, $c^{\prime}$ est que l'on ne peut rien entendre à et attendre de la théorie du cinéma dont nous avons hérité [26]si l'on se contente simplement de mettre à plat ce qu'elle doit [27] à la linguistique ou à la sémiologie sans en même temps indiquer ce que ce positionnement théorique engageait ailleurs: soit le rapport que cette $<<$ option >> linguistique allait entretenir avec les autres dimensions du langage et en particulier avec la rhétorique[28].Or à bien peser les choses, il apparaît que, bon an /pp 21-22/

mal an, la théorie du cinéma semble avoir essentiellement joué la relative $<<$ stabilité>> des relations qui existent entre grammaire et logique contre l'indécidabilité des relations qui régissent les échanges entre grammaire et rhétorique. L'une des principales caractéristiques de sa démarche a été de mettre à l'oeuvre la <<solidité>> des structures grammaticales et rhétoriques sans paraître même soupçonner parfois qu'il pût y avoir des contradictions ou des <<incompatibilités>> d'essence entre ces dernières[29]. Or ce n'est, nous semble-t-il, qu'en connaissance de cause, en tenant compte de cette difficulté théorique princeps, que l'on peut comprendre et évaluer l'émergence du concept de cinéma comme opérateur.

En effet, engager le film comme opérateur d'analyse, c'est, dans ces conditions, prendre acte de l'autre versant de ce qui est en jeu dans tout signifiant filmique, soit cette dimension qui, tout en étant encore langagière, ne sera plus passible d'une /pp 23-24/ analyse logico-grammaticale (que cette dernière soit de nature structuraliste ou générativiste). L'opérateur se plaira plutôt à jouer le statut $<<$ incertain>>, voire même aléatoire, du 
figural ou des tropes -- comme fonctions productrices d'effets textuels de lecture constamment <<ouverts >> - -- contre l'établissement de codes spécifiques, précipitant par là même une nouvelle économie de la lecture et de l'écriture. L'interprétation du signe filmique -- à présent abouté à des multiplicités plutôt qu'à des dichotomies --essayera moins de retrouver une structure (phrastique) ou de dégager un sens univoque sous les images et les sons que de produire un diagramme (un $<<$ volume $>>$ de sens) comme résultat d'une lecture stéréoscopique des différents régimes de signes qui caractérisent le texte filmique. C'est en ce sens que l'opérateur sera toujours une lecture et non un décodage: lecture de signes hétérogènes qui à leur tour renverront toujours à d'autres signes qu'il faudra interpréter à leur tour, ad infinitum [30].

En effet, ce qu'une analyse des grands textes de la sémiologie du cinéma montre à l'évidence, c'est la prédominance d'un modèle logico-grammatical très proche du modèle linguistique du Trivium classique, modèle où grammaire, rhétorique et logique entretiennent encore des relations qui sont hiérarchisées et relativement stables (non problématiques). Dans un tel modèle, les fonctions grammaticales et logiques du langage sont en parfaite $<<$ symbiose $>>$ et forment la précondition nécessaire et suffisante de tout $<<$ savoir $>>$ qui veut se présenter comme $<<$ science $>>$. Ici, $<<$ la continuité entre théorie et phénoménalisme est affirmée et préservée par le système lui-même $>>(R . T ., 14)$. Et de fait, tant que la théorie du cinéma a respecté ce principe, aucun problème ne s'est posé. C'est pourquoi il nous semble légitime d'avancer que c'est le renversement des $<<$ priorités $>>$ et des hiérarchies qui présidaient aux rapports entre la grammaire, la logique et la rhétorique, qui permet de comprendre le passage du cinéma de simple $<<$ objet >> d'analyse à celui d'analyseur, c'est-à-dire à ce que nous pourrions appeler un embrayeur (de jeux de langages) rhétorique. S'il fût un temps où l'alignement de la dimension rhétorique du cinéma sur les dimensions logiques et grammaticales du langage naturel a représenté une réelle percée théorique et a paru fournir une certaine stabilité aux $<<$ lectures du film $>>$ [31], le remplacement du modèle herméneutique d'interprétation (Bazin, Laffay, Mitry) par un modèle sémiotico-linguistique d'analyse par décodage n'a pas permis depuis de régler les problèmes: aussi raffiné soit-il, aucun décodage linguistique ou grammatical n'est à même de rendre compte de la multiplicité des dimensions sémantiques déterminantes qui sont mobilisées dans le moindre film. Tout film présente des éléments qui ne sont aucunement a-grammaticaux - -- mises en séquence narratives, résonances intertextuelles, phénomènes de réécriture ou citationnels, etc. -sans pour autant que leur fonction sémantique soit définissable ou analysable dans les termes d'une <<grammaire>>. [32]/pp 25-26/

Tant qu'elle était parvenue à maintenir ce $<<$ partage $>>$ et cette hiérarchie entre grammaire, logique et rhétorique, la sémiologie du cinéma a pu continuer de prospérer sans rencontrer d'obstacles majeurs. Tant qu'elle a pu maintenir le principe de cette partition et de ce partage des priorités, elle a pu apparaître comme la voie royale vers une science du langage cinématographique et du cinéma. Mais c'était compter sans la force 
disruptive du troisième terme de l'équation: la dimension rhétorique du cinéma. Et de fait, les difficultés n'ont commencé ici aussi à se faire sentir que $<<$ lorsqu'il n'a plus été possible d'ignorer la force épistémologique de la dimension rhétorique du discours; c'est-à-dire lorsqu'il n'a plus été possible de maintenir la rhétorique à sa place de simple supplément [<<adjunct $>>$ ] soit comme un simple ornement au sein de la fonction sémantique $>>$ (R.T., $14)$.

Mon hypothèse de $<<$ lecture $>>$ s'avère donc être la suivante: proposer le film comme opérateur, c'est faire retour à la $<<$ rhétoricité $>>$ du cinéma aux fins d'en mobiliser les ressources scripturales -- autant dire montagistes -comme $<<$ jeu de préfiguration du langage et de la symbolique disponible $>>$ [33] dans l'agencement des régimes hétérogènes de signes que tout film mobilise à chaque instant. De ce fait, qu'elle se présente comme une percée théorique ou un $<<$ coup de force>> épistémologique, la théorie du cinéma d'inspiration linguistico-sémiologique devra à son tour se révéler à son tour comme une forme spécifique de <<résistance à la théorie >>, mais cette fois non plus en tant qu'elle se refuse au concept ou au $<<$ théorétique $>>$ en général, mais en tant que son concept même (du cinéma) s'avère être lui-même le produit différé d'une $<<$ résistance $>>$ interne à la $<<$ déflection $>>$ rhétorique qui traverse de part en part le discours filmique. C'est en ce sens que, du point de vue de l'opérateur /pp 26-27/ filmique, elle se présentera en dernière instance comme une forme spécifique de $<<$ résistance $>>$ à la lecture. Pour reprendre ici les termes de Jacques Bessière, nous dirons que, comme pour l'oeuvre $(<<$ littéraire $>>)$, la $<<$ structure interrogative $>>$ du cinéma peut elle aussi être doublement caractérisée: $<<$ Structure d'interrogation du lecteur [du spectateur], structure d'interrogation de l'oeuvre [du film!] parce que [ici aussi] l'hypothèse d'une réalisation du langage $>>$ ne consiste plus dans l'abandon de l'oeuvre -- le film -- à $<<$ l'indifférenciation dans le langage $>>$, mais commandera plutôt de considérer que l'oeuvre [le film] $<<$ est bien ce qui participe d'un agon dans le langage et qui, par là, traite du partage qui instaure la création dans le discours $>>[34]$.

C'est ce genre de déplacement qui permet de comprendre certains des objectifs que s'était assignée la revue Hors-Cadre lorsque, dès son premier numéro, elle s'était donné pour tâche $\mathrm{d}^{\prime}<<$ user du cinéma comme d'un révélateur en le confrontant à des champs épistémologiques divers $>>$ (Hors-Cadre, I, 5.). Dans un tel contexte, <<si le cinéma fait office de miroir ici, ce sera pour les disciplines, les concepts, les démarches qui pourront s'y trouver réfractés $>>$ (Ibid.). Transformé en embrayeur (d'analyse), le cinéma invitera dorénavant à $<<$ travailler [avec lui] et non pas sur lui $>>$; il s'agira aussi, mais cela s'explique d'autant mieux que le cinéma est à présent assimilé à une rhétorique pratique, $<<$ non de l'éprouver comme ensemble textuel ou systématique, mais [de] faire jouer son aptitude à subvertir ou à confronter les textes, les systèmes, les scènes établies par ailleurs >> (Ibid., $5-6)$. 
Une telle conception impliquera plusieurs conditions nouvelles (pour la théorie du cinéma): 1) que le partage entre le langage (cinématographique) et le texte (filmique ou <<littéraire >>) ainsi que les présupposés théoriques sur lesquels repose une telle distinction soient radicalement remis en question; 2) cela impliquera aussi que le décodage grammatical (ou sémiologique) d'un texte laisse toujours un résidu d'indétermination -- une $<<$ déflection $>>$-- qui /pp 27-28/ devra être pris en charge non plus grâce à des moyens grammaticaux ou logico-sémantiques, mais au moyen d'une écriture renouvelée qui en refuse les garde-fous et qui n'hésite plusà s'aventurer sur le terrain glissant qu'ouvre la lecture rhétorique du texte par le film. Animée par la quaestio[35] rhétorique, l'analyse filmique devra dorénavant être à même de restituer au cinéma son dialogisme initial. La $<<$ révision >> que proposait la revue Hors-Cadre dès son premier numéro -- une révision que Marie-Claire Ropars-Wuilleumier présentait comme une $<<$ mise en crise $>>$ de la théorie du cinéma - -- conduira à formuler à nouveaux frais un concept d'écriture cinématographique[36] dont la $<<$ spécificité $>>$ consistera cette fois à faire du cinéma -- en tant que montage disjonctif d'éléments hétérogènes -- un $<<$ opérateur $>>$ privilégié de l'ébranlement des présupposés unitaires qui régissaient la linguistique et la sémiologie du cinéma: 1) le modèle du signe linguistique dont ces dernières se réclamaient; 2) la conception d'une énonciation filmique qui serait calquée sur les schémas de la communication; 3) la suprématie du logique et du grammatical sur le rhétorique (Modèle du Trivium ).

En tout cas, la conscience aiguè que l'on a désormais du jeu rhétorique des langages et des citations qui oeuvrent silencieusement dans l'entrecroisement des signes filmiques nous permet de comprendre que ce ne sera plus une <<grammaire >> (même renouvelée) du texte filmique ou une simple linguistique du signe qui devra être mobilisée pour la $<<$ lecture $>>$ et l'analyse du cinéma comme discours, mais une $<<$ syntaxe à relations contraires $>>$-- pour nous une rhétorique! - -- qui ne laissera dorénavant $<<$ courir le langage qu'en déroutant la parole $>>$ [37]. Opérateur de jeux et d'enjeux rhéto-/pp 28-29/ riques, l'opérateur filmique permettra de révéler sans $<<$ relève $>>$ les tensions latentes qui existent entre rhétorique et grammaire et, par là même, de relancer la lecture transversale des textes filmiques. Lire cinématographiquement se présentera dès lors comme un procès <<négatif $>>$ de déliaison au cours duquel la mise au pas grammaticale et/ou syntaxique du sens (commun) sera constamment défaite par la quaestio rhétorique[38] -- quaestio qui, nous espérons l'avoir montré un peu, ne pourra être prise en charge qu'à faire vibrer le sens en restituant à la lecture (du film) sa dimension de risque ainsi que sa nature d'événement.

Ce sont ces caractéristiques qui permettent de comprendre la nécessité où se sont trouvés des chercheurs venant d'horizons théoriques très différents[39] d'élargir le champ de leur investigation théorique à d'autres disciplines et d'en réévaluer le bien-fondé. Mais c'est qu'on ne pouvait pas faire remonter à la surface ce qui avait dû être refoulé pour tenir un 
discours monologique, sans avoir en même temps à reconsidérer la teneur de ce qui, il fut un temps, avait été considéré comme la théorie même ! Si notre hypothèse de lecture a quelque fondement, il devient alors nécessaire de concevoir l'émergence de l'opérateur filmique non plus seulement comme une simple tentative d'ajustement ou d'adaptation de $<<$ l'objet-cinéma $>>$ à tel ou tel <<acquis >> théorique nouveau -- linguistique, /pp 29-30/ psychanalytique, philosophique ou historique -- mais comme le signe ou peut-être mieux le symptôme d'une crise -- non plus régionale ou locale, mais générale -- de la théorie.

Déjà S.M. Eisenstein avait eu l'intuition de ce mouvement de généralisation de la crise (par le cinéma) lorsqu'au lieu d'essayer d'aligner le cinéma sur un langage ou une discipline déterminés, c'est plutôt au cinéma qu'il voulait confier la noble tâche de $<<$ relever $>>$ la déficience des disciplines qui s'occupaient des arts: peinture, photographie, architecture, ainsi que la langue elle-même! Pour Eisenstein, en effet, ce n'était point aux (autres) disciplines $<<$ scientifiques $>>$ de fournir au cinéma la $<<$ raison $>>$ de son bon fonctionnement, mais plutôt au cinéma -- pour lui un $<<$ langage $>>$, voire une langue aux potentialités infinies ! -- de fournir en $<<$ concepts $>>$ les discours sur les arts qui l'intéressaient. Et c'est ainsi que lorsqu'il convoquait la linguistique, la critique littéraire, l'histoire ou quoi que ce soit d'autre, c'est toujours à l'aune de la philosophie montagiste et $<<$ spontanément>> générativiste du cinéma qu'il essayait d'en traduire la complexité. À ses yeux, même le gros oeuvre de Marx ne perdait pas à être lu, et relu, à la lumière de la $<<$ dialectique $>>$ cinématographique.

Eisenstein avait clairement réalisé les potentialités <<opératoires >> du cinéma!

C'est, me semble-t-il, parce qu'ils ont renoué avec la même intuition[40], que les chercheurs, mentionnés plus haut, se sont retrouvés à interroger sur de nouvelles bases non seulement les présupposés théoriques qui déterminaient les $<<$ principes $>>$ de la recherche dans leur propre discipline, mais aussi les principes et présupposés qui conditionnent toutes les disciplines dont les objets venaient recouper -- serait-ce sous la forme de $<<$ codes non cinématographiques $>>$-- les leurs. Aujourd'hui, ni l'histoire, ni l'esthétique, ni l'analyse (textuelle) du cinéma ne peuvent se faire /pp $30-31$ / sans interroger les liens qui rattachent leurs <<objets $>>$ aux $<<$ objets >> des sciences de l'histoire, de l'esthétique ou de la critique en général.

\section{Brown University}

Department of French Studies

Providence - Rhode Island 02912 - USA 


\section{Surface Page d'Acceuil/Home Page}

[*] Le texte que l'on va lire est la reprise et le développement de l'exposé que j'ai fait lors de la table ronde organisée par le collectif de la revue HorsCadre pour son 10 e numéro consacré au thème: <<La recherche et l' "objetcinéma" >> , le 24 Juin 1991, à Paris.

[1] Je pense ici à l'écart qui sépare, sans toujours les opposer, d'une part, les travaux de critiques et/ou de théoriciens comme André Malraux, Maurice Merleau-Ponty ou Jean Mitry et, d'autre part, ceux de Christian Metz ou plus récemment de Gilles Deleuze ou Youssef Ishagpour. Du reste, le passage de la conception du cinéma comme objet d'analyse au cinéma comme opérateur d'analyse ou, plus radicalement, comme analyseur, me semble s'être $<<$ négocié $>>$ à partir du traitement théorique de cet écart. Ce n'est, en effet, qu'en connaissance des attendus théoriques des $<<$ travaux $>>$ de chercheurs qui les ont précédés que les propositions sur le cinéma comme <<opérateur>> d'analyse ont pu être formulées positivement: cf HorsCadre, No 1 (Analectures), pp. 7-9.

[2] Je pense ici aux réflexions de Gilles Deleuze dans Cinéma II. L'ImageTemps, et en particulier à ce qui est articulé dans le chapitre intitulé $<<\mathrm{La}$ pensée et le cinéma $>>$ : $<<$ Le fait moderne, c'est que nous ne croyons plus en ce monde. Nous ne croyons même plus aux événements qui nous arrivent, à l'amour, la mort, comme s'ils ne nous concernaient qu'à moitié. Ce n'est pas nous qui faisons du cinéma, c'est le monde qui nous apparaît comme un mauvais film. À propos de $<<$ Bande à part $>>$, Godard disait: $<<$ Ce sont des gens qui sont réels, et c'est le monde qui fait bande à part. C'est le monde qui se fait du cinéma. C'est le monde qui n'est pas synchrone, eux sont justes, sont vrais, ils représentent la vie. Ils vivent une histoire simple, c'est le monde autour d'eux qui vit un mauvais scénario >>.>> Deleuze, Ibid, p. 223. Les italiques sont de moi.

[3] Je pense ici à des gens aussi différents que Bela Balàsz ou Kracauer, par exemple, ou à des critiques comme Élie Faure ou André Bazin; ou encore à Maurice Merleau-Ponty ou André Malraux.

[4] À ce propos, voir les articles décisifs que Christian Metz a consacrés à ces questions dans ses Essais sur la signification au cinéma, Tome II, Nincksieck (Esthétique), 1972. Revoir en particulier, <<Une étape dans la réflexion sur le cinéma $>>$ (1964), pp. 13-34 et $<<$ Problèmes actuels de théorie du cinéma >> (1966), pp. 35-96. 
[5] Ou $<<$ André Bazin $>>$ ou encore $<<$ André Malraux $>>$ ou même $<<$ Jean Mitry $>>$ !

[6] Peut-être vaudrait-il mieux parler ici de $<<$ moment $>>$ - au sens d'une certaine vulgate hégélienne - - plutôt que d'<<époque>> dont on pourrait réserver la charge sémantique d'Epochè - au sens, heideggerien cette fois, de suspension et d'arrêt - pour caractériser ce qui se produira au moment où le cinéma ne se pliera plus aussi docilement aux méthodologies $\mathrm{d}^{\prime}<<$ application > > et se transformera en instrument d'analyse inouï. À ce propos, je renvoie bien évidemment aux travaux de Deleuze sur le cinéma, Cinéma I et Cinéma II, mais aussi à ceux de Marie-Claire RoparsWuilleumier. Voir en particulier, Le Texte Divisé, Paris, P.U.F (Ecriture) 1981 et Écraniques. Le Film du texte, P.U.L. (Problématiques), 1990, où il est établi clairement que <<travaillant simultanément sur des objets filmiques et des concepts éprouvés dans le champ littéraire, on esquisse ici le trace d'une zone frontalière, où la réflexion circule entre la littérature et le cinéma, la théorie et l'analyse, et cherche dans les films un surplus critique pour une étude du texte>> (<<Avant-propos>>, in Le texte divisé). C'est moi, R.B., qui souligne.

[7] Je renvoie ici aux travaux essentiels de Christian Metz et tout particulièrement à ses Essais sur la signification sur le cinéma, (Tome I et II), Klincksieck et bien sûr à l'impressionnant Langage et Cinéma, Paris, Editions Albatros (Ça/Cinéma), 1977. De fait, ces textes sont ceux qui ont définitivement arraché le cinéma à $l^{\prime}<<$ usure >> idéologique et psychologique dont il avait été l'objet pour le transformer en langage et bientôt en texte à...produire!

[8] Vocation du cinéma, Ed. Gonthier (Bibliothèque Médiations), 1964, p. 69.

[9] $\mathrm{L}^{\prime}<<$ époque>> que je tente de pointer ici ne doit déjà plus rien à Bazin et commence à se démarquer par rapport à $l^{\prime}<<$ âge $>>$ sémiologique du cinéma. Cette <<époque>> tire résolument le cinéma vers une $<<$ logistique de la perception $>>$ qui est plus proche des réflexions d'un Heidegger sur $l^{\prime}<<$ essence de la technique $>>$ que des considérations encore bien $<<$ humanistes $>>$ d'un Malraux sur l'<<art $>>$ et nos $<<$ musées imaginaires $>>$ ! Cf Paul Virilio, $<<$ Guerre et Cinéma 1. Logistique de la perception>>, Cahiers du cinéma, Paris, Éditions de l'Étoile, 1984.

[10] Paul Virilio, La Machine de Vision, Galilée, p. 126.

[11] Paul Virilio, Ibid., pp. 127-128.

[12] Je forge ici ces deux expressions non par un goût particulier pour les néologismes, mais par la nécessité où je me retrouve de qualifier la nature des déplacements qui ont précédé et conditionné l'émergence du concept de cinéma comme opérateur: concept que je rattache donc moins à un développement linéaire de la théorie du cinéma qu'à une $<<$ logique>> paradoxale de renversement des perspectives. 
[13] Gilles Deleuze, $<<$ La pensée et le cinéma $>>$ et $<<$ Cinéma, corps et cerveau, pensée>>, in Cinéma II, Ibid.

[14] Christian Metz, <<Le perçu et le nommé>>, in Essais de Sémiotique, Nincksieck (Esthétique), 1977, pp. 130 et ss. <<I1 faut d'abord délimiter l'objet même de la recherche, écrit Metz, et le délimiter sur ses deux flancs. Les codes de nomination iconiques ne mettent pas en rapport le tout du langage et le tout de l'image; leur étude ne doit pas prétendre à épuiser la vaste question des liens entre le perspectif et le linguistique, mais se concentrer au contraire sur un des niveaux pour tenter de mieux l'éclairer >> (p. 133). C'est moi, R.B., qui souligne.

[15] En ce sens, ni Faure, ni Bazin, ni Mitry ne peuvent être considérés comme des penseurs dogmatiques. Ils se sont eux-mêmes présentés comme des essayistes qui aimaient le cinéma et qui désiraient le prendre au sérieux. Ce qui peut nous apparaître aujourd'hui comme dogmatique ou doctrinale est plutôt l'effet d'une lecture rétrospective! Par contraste, tous les théoriciens modernes peuvent au contraire être dits $<<$ dogmatiques $>>$ en ce que leurs $<<$ théories $>>$ se veulent fondées en théorie et de ce fait discutées et critiquées en bloc.

[16] Au sens que G. Vattimo donne à ce mot.

[17] Paul de Man, Resistance to Theory, Minneapolis, University of Minnesota Press, p. 6. Dorénavant, je ne me référerai plus à ce texte que par les initiales R.T. Je voudrais aussi signaler que je dois une certaine orientation de mon propos aux thèses qui sont avancées par De Man dans le texte susmentionné.

[18] Je me réfère ici à ce que Paul de Man écrivait à propos de la critique littéraire: <<Literary theory can be said to come into being when the approach to literary texts is no longer based on non-linguistic, that is to say historical and aesthetic considerations or, to put it somewhat less crudely, when the object of discussion is no longer the meaning or the value but the modalities of production and of reception of meaning and of reception of meaning and of value prior to their establishment - the implication being that this establishment is problematic enough to require an autonomous discipline of critical investigation to consider its possibility and its status". (R.T., 7) C'est moi qui souligne.

[19] Hors-Cadre, No.7 ( $<<$ Théorie du cinéma et crise dans la théorie >>), Hiver 1988-1989, et en particulier les articles de Pierre Bayard: <<Et pourtant...Théorie et sujet du paradoxe>>; Joan Copjec: $<<$ Le sujet orthopsychique: théorie du film et réception de Lacan>>; Jacques Aumont: $<<$ Crise dans la crise $>>$; Réda Bensmaïa: $<<$ L'intérêt de la crise $>>$.

[20] Une <<autorisation>> - - est-il nécessaire de le préciser?- dont il ne s'agit pas de juger la valeur heuristique des $<<$ résultats $>>$ auxquels elle a donné lieu, mais d'interroger les $<<$ titres $>>$. En effet, comme j'essaierai de le montrer plus clairement plus loin, il ne fait aucun doute que le type de questionnement qui affecte la $<<$ théorie $>>$ du cinéma aujourd'hui serait impensable sans le travail de déchiffrage et de clarification qui a été mené 
par les différentes approches sémiologiques du cinéma. On voit bien aujourd'hui que ce n'est qu'au prix de $l^{\prime}<<$ arrachement $>>$ du cinéma à la critique idéologico-esthétique qui l'avait pris en charge pendant plus de trois décades que la réflexion dont il est devenu l'<<objet $>>$ est devenue possible! En ce sens, les travaux d'un Souriau et bien entendu ceux d'un Metz ont joué et continuent de jouer un rôle essentiel indépassable.

[21] Paul de Man, Ibid., p. 9. En ce qui concerne l'impact de l'intervention de la linguistique $<<$ à travers champs disciplinaires $>>$, on consultera avec intérêt le beau livre de Thomas Pavel, Le Mirage Linguistique. Essai sur la modernisation intellectuelle, Paris, Minuit, 1988 et en particulier $<<$ Technologie et Régression $>>$, pp.31 et ss, et $<<$ Les comportements intellectuels discrétionnaires $>>$ où l'on peut lire en page $178:<<[\ldots]$ au fond, soit qu'avec ferveur on nous propose quelque théorie sémiotique du sens, soit que d'un ton ironique on démonte le vain jeu des différences, c'est toujours à l'identification du sens au signe qu'on a affaire, c'est toujours la réduction de l'activité au schématisme linguistique, et le remplacement de l'intention par le jeu indéterminé des coutumes qui dès avant le début de la démonstration en pipent les dés >>. C'est moi qui souligne.

[22] Je ne peux ici que renvoyer au travail magistral de Jacques Bessière dans son Dire le Littéraire. Points de vue théoriques, Liège-Bruxelles, Pierre Mardaga Éditeur, 1990, dont je ne citerai que ces quelques phrases pour illustrer mon propos: <<Des théories contemporaines du littéraire, de ce qu'elles disent et contredisent, de leurs dialogues et de leurs oppositions, il pourrait se construire une manière de rhétorique, à la façon dont Paulhan a noté, dans Les Fleurs de Tarbes, les inconséquences et, par là, l'unité rhétorique de la critique littéraire [...]. Dire le formalisme, l'herméneutique, la pragmatique littéraire, c'est entreprendre de marquer en quoi le littéraire peut être ultimement inconditionnel, exemplairement le lieu de la réalisation discursive et de la régulation communicationnelle, et inévitablement constater que cette inconditionnalité reste inséparable, dans sa notation, du constat que le littéraire échappe à tout jugement d'identification et qu'il peut encore, par là, se définir comme un inconditionnel commun - par quoi s'expliquent l'apparentement du littéraire et l'éventuelle dénégation de tout effort critique pour OBJECTIVER le littéraire.>> (pp. 55-56). Les majuscules sont de Bessière.

[23] Comme attention à la référence plutôt qu'au référent, au procès de signification plutôt qu'à l'intuition.

[24] < <Ainsi la linguistique, écrivait Christian Metz dans un texte fondateur, grâce à son analyse de la langue, éclairant au départ ce que le cinéma n'est pas, mène insensiblement à entrevoir ce qu'il est, et ce, dans le mouvement où elle se couronne elle-même d'une translinguistique (sémiologie) $>>$, in $<<$ Cinéma: langue ou langage?>>, Essais, p. 87.

Il faudra attendre les travaux de Gilles Deleuze pour que le bien-fondé et le caractère d'évidence des présupposés qui étaient mis en jeu par l'ouverture metzienne se trouvent relevés. Gilles Deleuze, Cinéma II,$<<$ Récapitulation des images et des signes $>>$, pp. 38 et ss. En particulier p. 38: $<<$ Substituant à l'image un énoncé, [Metz] peut et doit lui appliquer 
certaines déterminations qui n'appartiennent pas exclusivement à la langue, mais qui conditionnent les énoncés d'un langage, même si ce langage n'est pas verbal et opère indépendamment d'une langue $[. .]>.>$. Et un peu plus loin, p. 39: <<La sémiologie du cinéma sera la discipline qui applique aux images des modèles langagiers, surtout syntagmatiques, comme constituant un de leurs $<<$ codes $>>$ principaux. On parcourt ainsi un cercle étrange, puisque la syntagmatique suppose que l'image soit en fait assimilée à un énoncé, mais puisque c'est elle aussi qui la rend en droit assimilable à l'énoncé $[\ldots]>>$.

On se reportera aussi avec intérêt à l'article de Marie-Claire RoparsWuilleumier, $<<$ Christian Metz et le mirage de l'énonciation $>>$ où c'est la problématique linguistique de l'énonciation et non plus seulement de l'énoncé qui est questionnée au profit de l'opérateur-cinéma: <<L'ordre de la langue sert ainsi tour à tour, de contre-exemple restrictif qui amplifie a contrario l'expansion du film, et de réserve critique, destinée à maintenir intacte, ailleurs, la pureté de la loi. C'est cet ailleurs linguistique [...] qui constitue, peut-être, une variante du mirage énonciatif [...]. Prendre le parti de l'impureté cinématographique, ce serait, en retournant la perspective, adopter le point de vue de la voix dans l'écoute de la vision. Si l'on accepte de modifier ainsi l'angle d'approche, le cinéma se révèle un remarquable opérateur pour mettre en scène les équivoques de l'énonciation [...]>>, in $<<$ Christian Metz et la théorie du cinéma>>, Iris, No.1O, Avril 1990, p. 109. C'est moi, R.B., qui souligne.

Plus loin, j'espère pouvoir montrer que ces <<équivoques $>>$ tiennent au $<<$ refoulement $>>$ de la dimension rhétorique qui travaille toute écriture et à plus forte raison, l'écriture filmique.

[25] Roger Odin, <<Christian Metz et la linguistique>>, in Iris, No 10, Avril 1990, pp. 81 et ss.

[26] Je parle de la théorie du cinéma d'inspiration linguistico-sémiologique.

[27] À ce propos, les temps sont peut-être venus où la question du transfert théorique d'une discipline à une autre - ici de la linguistique et de la psychanalyse au cinéma, par exemple - devrait enfin pouvoir être posée. Ce geste serait le meilleur indice de la $<<$ maturation $>>$ de la théorie concernée. Cette $<<$ question $>>$ nous semble avoir été directement soulevée dans le No.7 de Hors-Cadre. Voir plus haut, note 19.

Pour ce qui a trait à la problématique de la dette (théorique) et de la culpabilité qu'elle engendre du fait du caractère $<<$ nécessairement agonistique de toute interprétation $>>$, voir Samuel Weber, $<<$ The debt of criticism: Notes on Stanley Fish's Is there a text in this class? $>>$ et $<<$ The debts of Deconstruction and other related assumptions > >, in Institution and Interpretation, Minneapolis, University of Minnesota Press (T.H.L. series), Volume 31, pp. 33-39 et 102-131.

[28] Je tiens à préciser que par rhétorique, je ne me réfère pas ici à l'étude des tropes et des figures ou à la $<<$ science > > de l'éloquence et de la persuasion, mais à l'étude du discours lorsqu'il est abordé, par des critiques comme Kenneth Burke ou Paul de Man, à partir de ces petites déhiscences 
ou glissements du sens qui rendent problématiques la confiance que l'on a généralement en la toute puissance de la dimension logico-grammaticale du langage.

Je prends pour guide ici la conception offerte par un Burke lorsque, concernant le discours littéraire, il avançait l'idée que si ce discours était fait d'agencements logiques et grammaticaux, il fallait toujours tenir compte en même temps de ces $<<$ déflections $>>$ [ $<<$ deflections $>>$ ] - assimilées par lui au $<<$ déplacement $>>$ freudien et qu'il définissait comme $<<$ ces petites distorsions, préjugés, penchants $[<<$ bias $>>$ ] ou même ces erreurs inintentionnelles $>>$ - qui venaient constamment en contaminer la transparence. Ainsi définie comme la base rhétorique du langage et comme une dimension inhérente à son fonctionnement, la $<<$ dénection [est] conçue comme une subversion dialectique de la consistence des liens qui unissent le signe et le sens qui sont à l'oeuvre dans les structures grammaticales $>$ >. D'où la nécessité, pour Burke, de distinguer radicalement la grammaire de la rhétorique. Dans une telle perspective, parler de (dimension ou d'effets) rhétorique(s) ne consistera plus à simplement repérer et bientôt opposer un sens littéral à un sens figuré (sens restreint de la rhétorique), mais plutôt à mettre en évidence $<<$ l'impossibilité de décider par des moyens purement grammaticaux ou linguistiques laquelle des deux significations $[<<$ meanings $>>$ ] - des significations qui peuvent être radicalement incompatibles! - prévaut $>>$. Et en ce sens, la rhétorique dont nous nous prévalons ici sera celle qui <<suspend radicalement la logique et ouvre aux possibilités vertigineuses de l'aberration référentielle $>>$. Kenneth Burke, $<<$ The concept of Literature >> , in Literary theory and Structure: Essays in Honor of William K. Wimsatt, New York, Frank Brady, John Palmer and Martin Price, eds, 1972, et Paul de Man, <<Semiology and Rhetoric >>, in Allegories of Reading. Figural language in Rousseau, Nietzsche, and Proust, New Haven and London, Yale University Press, 1979, pp. 7-8. C'est moi, R.B., qui souligne. On se reportera aussi avec intérêt au très beau livre de Laurent Jenny, La Parole singulière, Berlin et Paris, 1990 et en particulier $<<$ Passage de la catachrèse $>>$, pp. 35 et ss où l'on peut lire, entre autres, ceci qui éclaire bien notre propos sur l'intervention du rhétorique /pp 22-23/ comme <<événement>> hétérogène à la grammaire et à la syntaxe: $<<$ L'événementialité du figural [...] ne se laisse pas saisir sans ombre portée qui est sa disparition dans l'inactuel. Car le figurat ne cesse d'apparaître et de disparaître malgré l'effort solidaire des grammairiens pour définir des normes et des rhétoriciens pour objectiver des types d'écart. Chacun sent pourtant que les grammaires et les rhétoriques sont aussi impossibles que nécessaires. Impossibles parce que les limites qu'elles tracent (à la norme, à l'écart - c'est-à-dire à l'événement) peuvent à tout moment être déplacées par l'événement même, c'est à dire par une situation de parole réelle où les interlocuteurs engageront une représentation de la langue autre que celle qui leur est assignée. Nécessaires parce qu'il faut au moins provisoirement s'entendre sur la forme de la langue si l'on veut parler, et faire de la parole le cadre de déplacements et de réévaluations de cette forme $>>$. Plus loin (pp. 36-37) L. Jenny en arrive <<spontanément>> à parler $\mathrm{d}^{\prime}<<$ opérateurs $>>$ rhétoriques.

[29] Et ce, malgré les <<signaux>> ainsi que les appels à la prudence qui avaient été lancés par Metz dès Le Signifiant imaginaire. 
[30] Charles Sanders Peirce, Écrits sur le signe, Paris, Le Seuil, 1978, pp. 120 et ss. Et p. 126: < < [En bref, un signe est] tout ce qui détermine quelque chose d'autre (son interprétant) à renvoyer à un objet auquel lui-même renvoie (son objet) de la même manière, l'interprétant devenant à son tour un signe et ainsi de suite ad infinitum $>>$. C'est cette espèce de $<<$ dérive $>>$ folle que l'opérateur-cinéma prend en charge. On pourrait à ce propos mettre au compte du cinéma comme $<<$ opérateur $>>$ tout ce que dit Paul de Man de ...Peirce: $<<$ Peirce nomme ce procès par lequel $<<$ un signe donne naissance à un autre $>>$ pure rhétorique en tant que celle-ci se distingue de la pure grammaire qui postule la possibilité d'un sens [meaning] diadique non problématique et de la logique qui elle, de son côté, postule la possibilité de la vérité universelle des significations $>>$. (Paul de Man, Allegories of Reading, p. 9)

[31] Jean Collet, Michel Marie, Daniel Percheron, Jean Paul Simon, Marc Vernet, Lectures du Film, Paris, Albatros, 1976 (réédité en 1980).

[32] Paolo Valesio, Novantiqua, Rhetorics as a Contemporary Theory. Bloomington, Indiana University Press, 1980: <<La narratologie est en même temps trop large et trop limitée comme technique descriptive pour être directement utile à l'élaboration d'une théorie de la rhétorique. Trop large, parce que (...) ses unités sont généralement plus vagues que les unités que l'analyse linguistique aime à mobiliser; et trop limitée, du fait qu'elle se restreint à l'analyse de la narration, alors que nous nous intéressons à la structure de tout type de discours... >> (p. 9).

[33] Jacques Bessière, Ibid, pp. 309 et ss.

[34] Jacques Bessière, Ibid.

[35] Voir ici même le développement qui est donné à la quaestio par J. Bessière.

[36] D'inspiration grammatologique et non plus simplement logicogrammatical ou représentatif.

[37] Marie-Claire Ropars-Wuilleumier, Ecraniques, Ibid, p. 13.

[38] Pour que les choses soient claires, nous dirons qu'il y a $<<$ question rhétorique $>>$ lorsque nous avons affaire à la $<<$ symbiose $>>$ entre une structure grammaticale et une structure rhétorique et que du fait de cette symbiose, il devient impossible de décider s'il faut opter pour un sens $<<$ littéral $>>$ ou un sens $<<$ figuré $>>$.

[39] Je pense ici, bien évidemment, au collectif de la revue Hors-Cadre, mais aussi à des chercheurs comme Gilles Deleuze, Youssef Yshagpour, Jean-Louis Scheffer ou Serge Daney pour ce qui a trait directement au cinéma. Mais il est évident que la $<<$ rhétoricité>> filmique n'a pas manqué d'engager des chercheurs d'autres champs disciplinaires dans le mouvement de reprise et de réévaluation des présupposés théoriques que nous avons tenté de décrire ici. 
[40] Dans les conditions historico-théoriques que nous avons tenté d'analyser dans cet article: suprématie de la science linguistique, montée de l'<<idéologie $>>$ structuraliste, etc. 